\title{
Developing Disposable Hair Washing Pad for Bedridden Patients Using Mixed Methods Research
}

\author{
Sachiko Makabe ${ }^{1}$, Katsushi Maeda ${ }^{2}$, Sayaka Izumori ${ }^{2}$, Emiko Konno $^{3}$, Yayoi Sato ${ }^{3}$, Nana Yoshioka ${ }^{3}$, \\ Hideko Shirakawa ${ }^{3} \&$ Kenji Ando ${ }^{2}$ \\ ${ }^{1}$ Clinical Nursing Department, Akita University, Japan \\ ${ }^{2}$ R\&D-Development Research-Sanitary Product Research, Kao Corporation, Japan \\ ${ }^{3}$ Akita University Hospital, Japan \\ Correspondence: Sachiko Makabe, Clinical Nursing Department, Graduate School of Health Sciences, Akita \\ Univeristy, Akita city, 010-8543, Japan. Tel: 81-18-884-6539. E-mail: 1smakabe@gipc.akita-u.ac.jp
}

Received: April 5, 2019 Accepted: April 25, 2019 Online Published: April 29, 2019

doi:10.5539/gjhs.v11n6p70 URL: https://doi.org/10.5539/gjhs.v11n6p70

\begin{abstract}
In Japan, diapers are often used to wash bedridden patients' hair by placing the diaper under the patient's head for ease of use and efficiency. However, using diapers for the head is an ethical problem as diapers are originally used for elimination care. Developing better equipment to wash hair is necessary to comfort patients and reduce nurses' workload. Our industry-academia-clinical collaboration team developed a disposable absorbent pad particularly for washing patients' hair in bed. This study aimed to evaluate this pad developed for bedridden patients. The trial was conducted in five departments at a university hospital between May and August 2016. The post-trial cross-sectional survey for nurses contained demographic data, evaluation of the pad with rating score as quantitative data, free comments as qualitative data, and comparison with diaper used experience. As this study involved development of an equipment and thus to facilitate data triangulation, mixed methods were used. The results revealed that 36 nurses participated (90\% response rate). Most were in their 20s (69\%). The overall evaluation was "good" (91\%). Good water absorption, no water leakage, and easy usage were reported. In a comparison with diapers using experience, the majority preferred the developed pad (81\%). Better structure, usage, and resolution of ethical issues were also confirmed in a comparison with diapers. The developed disposable hair washing pad is an efficient tool for nurses to wash bedridden patients' hair. As it is disposable, infections are also controlled well. Further manufacturing aspects need to be considered for mega production.
\end{abstract}

Keywords: hair washing in bed, disposable absorbent pad, mixed methods research, fundamental care, nursing equipment development, industry-academia-clinical collaboration

\section{Introduction}

\subsection{Background of Hair Washing in Bed}

Bedridden patients' scalp and hair are easily stained, and eccrine gland secretions due to sympathetic nerve predominance cause discomfort. A hair wash does not merely retain cleanliness but also helps patients feel better and recover (Dougherty \& Lster, 2004). Hair washing is associated with mind-body-spirit connection for healing, because distress could affect health, both psychological and physical (John \& Gross, 2004; Roberts, Levenson, \& Gross, 2008; Schlatter \& Cameron, 2010). Consequently, washing hair in bed is an essential aspect of care (Sartini et al., 2018). Fundamental care in critical care settings is recognized as a complex activity crucial for patients' safety, recovery, and wellbeing (Feo \& Kitson, 2016). Research on hair washing is limited compared to that on bed bath (Groven et al., 2017). In Japan, diapers are often used as equipment to wash bedridden patients' hair by placing them under the patients' head (Nakai et al., 2009; Tamura et al., 2013). Reasons for using diapers are convenience, easy disposability after use, reduced time consumption, and physical comfort of patients. However, using diapers for the head is an ethical problem, because they are originally used for elimination care (Nakai et al., 2009). Standardization/efficiency is essential for the highly demanding hospital environments (Friganovic et al., 2017). Disposability is also valuable for infection control.

In not just hospital but also home care settings in Japan, diapers are often used for washing bedridden patients' hair (Nakai et al., 2009; Tamura et al., 2013). In the super aging and depopulated society of Japan, the proportion of 
aging population will reach $48 \%$ in 2025 (Chen et al., 2016). Washing bedridden patients' hair needs to become an easy for of care for everyone. Moreover, futon (sleeping on the floor) is more popular than sleeping on the bed, especially in Asian countries (Makabe et al., 2015). The disposable hair washing pad for bedridden patients is useful owing to a height that is low to facilitate for water drainage. Because of all these reasons, the development of disposable hair washing pad for bedridden patients is crucial.

\subsection{Challenges of Existing Hair Washing Sheet}

Only one type of hair washing sheet is available in the market in Japan. Sato et al. (Sato, Kodo, Fukushi, \& Isomoto, 2014) have reported the evaluation of this hair washing sheet. Challenges of using the sheet include 1) poor fit around the back of the neck, 2) less water absorption than diapers, and 3) water easily accumulates behind the head. The sheet is flat and does not gather around behind the neck; only 2 liters of water can be absorbed. The size is $900 \mathrm{~mm} \times 600 \mathrm{~mm}$, which is relatively large, and the head is also fixed on one particular part of the sheet. Consequently, another type of disposable hair washing pad to meet bedridden patients' needs must be established.

\subsection{Benefit of Industrial-Academia-Clinical-Collaboration}

Reports on the development of nursing equipment are limited. Industry-academia-clinical collaboration is important (Tsuruya et al., 2018) for industry to be more academic, for academic to be more citizen ship, and for clinical to be more development mind benefiting on patient. For development of equipment, a different specialty team is required (Murayama, 2016). It is essential to develop specialized functions that can be broadly applied (Tsuruya et al., 2018). Our industry (disposable absorbent pad specialty)-academia-clinical collaboration team brainstormed ideas together and developed a disposable absorbent pad particularly for washing bedridden patients' hair.

\subsection{Why Mixed-Methods Research}

Mixed methods research is ideal for a development study (Fetters, Curry, \& Creswell, 2013). Our study involved quantitative data to capture an overview confirming acceptability of the developed pad. Qualitative data involved users' opinions to understand challenges in detail, for further development. Integration of these dual types of data allowed a holistic evaluation by triangulation. The study aimed to evaluate the developed disposable hair washing pad for bedridden patients in terms of its usage and ethical aspects.

\section{Method}

\subsection{Development Process}

The lead researcher presented the idea of disposable hair washing pad for bedridden patients in a "talk session on goals" at the university to which she/he belonged. Industrial-academia department from the university supported the connection with the top company research department (specialized in disposable absorbent pad) in Japan. Then this research project was launched in May 2015. At a university hospital, our project team observed the actual bedridden patients' hair washing methods and interviewed nurses about challenges. We also conducted a pre-survey to identify the department where bedridden patients' hair washing was needed the most, at the university hospital setting of this study. The already marketed hair washing sheet was also tested for structural and usage qualities. For a year, numerous prototypes were produced throughout the process of testing on simulated patients (nurses or nursing students) concerning water absorption level, water leakage, size, design, usage, and patient comfort.

The pad (Figure 1) was finally developed after saturation of any perspectives. This pad had a simple rectangular shape $(420 \mathrm{~mm} \times 1040 \mathrm{~mm})$ with pale pink check pattern. It had a maximum absorption capacity of 4 liters of water and enough strength to hold polymer. It could be fit with gather shape at the back of the neck to avoid water leakage. Washing hair begins from one end, moves to the middle, and then ends including drying, at the other end of pad. The nurse needs to lift the head up slightly and slide the pad (Figure 1). Figure 1 also includes an instruction manual for nurses. Patent was applied for at Japan patent office in 2017 (Makabe et al., 2017b) and it was registered in 2018.

\subsection{Study Design}

The trial of the pad was conducted, in addition to a cross-sectional survey using mixed methods with a convergent design. Mixed methods were used because this is a development study and results needed to be triangulated (Creamer, 2018). Figure 2 shows the procedural diagram of the convergent study design. Both quantitative and qualitative data were collected at the same time. Dual data analyses were conducted separately, and then the qualitative results were merged with the quantitative result. Interpretation appears integrative using joint display. 


\subsection{Study Setting and Participants}

From May to August 2016, the top five units implementing high-frequency bedridden patients' hair washing, including intensive care units at a university hospital, were used as the setting. The sample size was aimed to include 40 nurses in total (approximately 8 nurses per unit) calculated from a reasonable sample size based on units' hair washing needs. After using the pad, the nurses answered the questionnaire. There were no inclusion or exclusion criteria for nurses. For patients, inclusion criteria were 1) needing hair washing in bed, 2) aged over 18 years, and 3) being of any sex. Exclusion criteria were 1) having an excessively high-risk medical condition that is life threatening and 2) not receiving consent even from a legally authorized representative.

\subsection{Questionnaire Contents}

Demographic data for nurses included age, nursing experience years, and department type. For patients, they included sex, age, hair length, period since last wash, and water volume used.

For nurses, an overall evaluation was sought, with responses categorized as "very good," "good," "neither," "bad," and "very bad" (5 levels) followed by a free comment for reasons. Subsequently, details of structural and usage aspects were asked. Structural aspects included "water absorption," "shape," "gathering behind the neck," and "design of pattern/color." Response categorizations were "very good," "good," "neither," "bad," and "very bad" (5 levels). Usage aspect included "easy to wash," "water leakage," "water spreading," “easy to prepare," and "easy to clean" with response categorization of "yes" or "no." Diapers were not used for our trial due to ethical concerns, but all nurses had experience of using diapers for hair washing in the past. The question comparing the developed pad to diapers only included the response categorization of "very good," "good," "neither," "bad," and "very bad" (5 levels) followed by free comment for reasons. Feeling and experience of using a diaper as hair washing equipment were also asked with response categorization of "yes" or "no."

For patients, neck comfort was asked with response categorization of "very comfortable," "comfortable," "neither," "unpleasant," and "very unpleasant" (5 levels) followed by free comments.

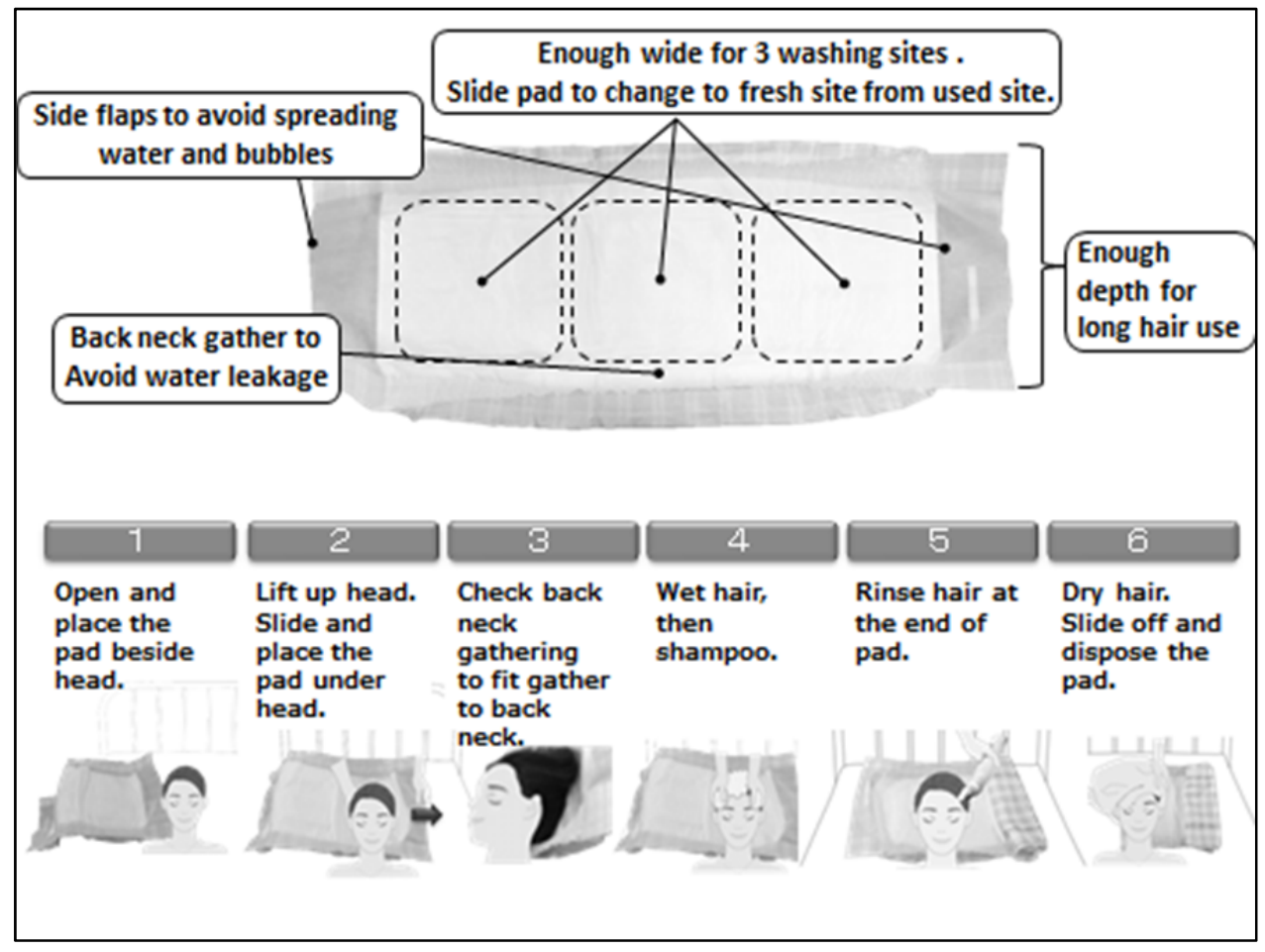

Figure 1. Disposable hair washing pad for bedridden patients and instruction manual 


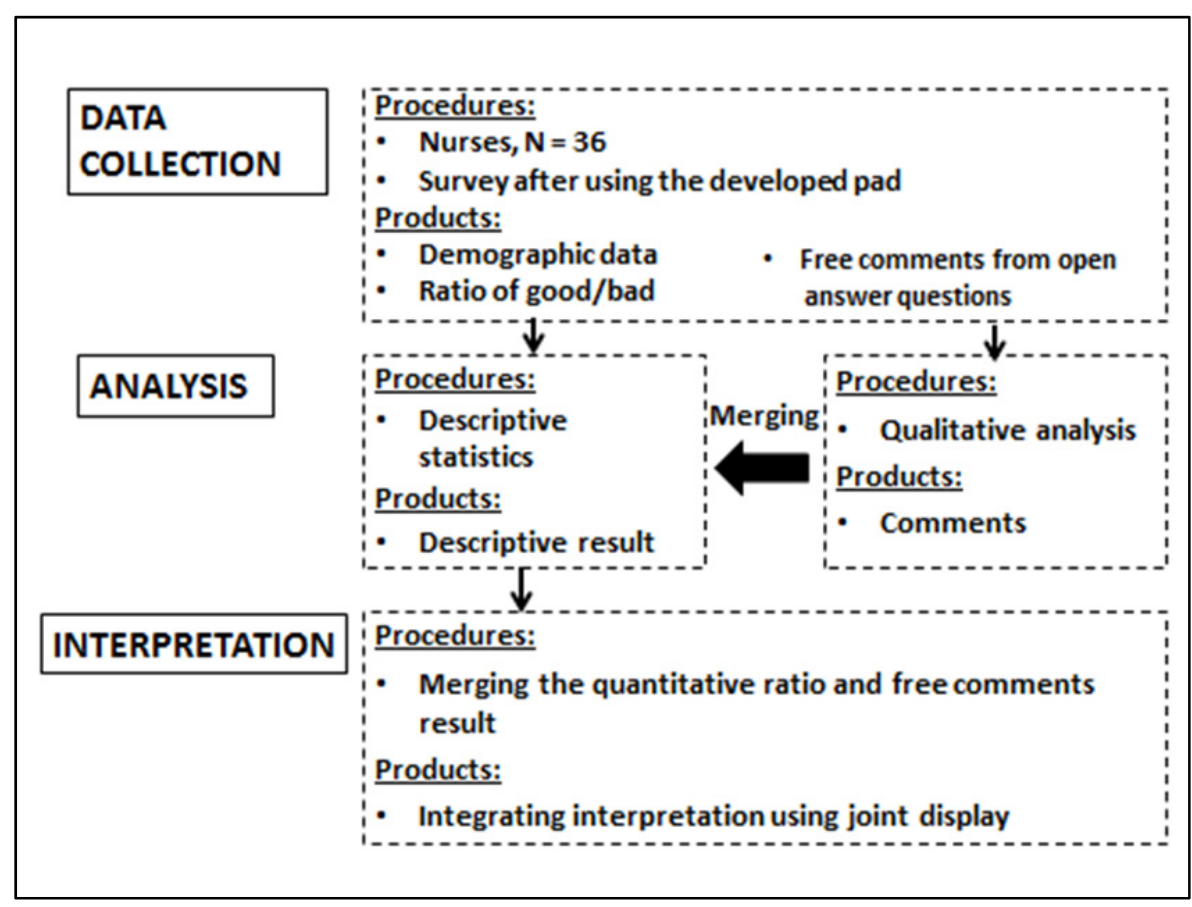

Figure 2. Procedural diagram of the convergent study design

\subsection{Statistical Analyses}

Demographic data were simply aggregated. For the overall evaluation from both nurses and patients, the proportion was calculated, and then free-comment qualitative data were analyzed using qualitative analysis. For the detailed evaluation, proportion was calculated. For the comparison with diaper, proportion was calculated, and then free comments were analyzed. Result of evaluations and comparisons were presented as a joint display by triangulating quantitative and qualitative data (Creamer, 2018).

For quantitative data, JMP 13 (SAS institute) was used. For qualitative data, three researchers, including non-project members, analyzed the qualitative data independently. The industrial co-researcher team with three members analyzed the qualitative data independently. Then the analyzed qualitative results were discussed for consensus between members. This method was used for trustworthiness (Korstjens \& Moser, 2018) to improve the qualitative analyses.

The methodology was presented (Makabe et al., 2017a) and discussed at an international mixed methods research conference in 2017 and guidance was received from experts in mixed methods research methodology.

\subsection{Ethical Considerations}

The study was ethically approved (registration number 1488). It was also registered as a clinical trial at UMIN, the university hospital medical information network in Japan (registration number UMIN000022100).

Nurses received face-to-face explanation and not just a letter, about the study aim and methods, which included the following: 1) their privacy was strictly protected;2) refusal to participate would not influence their work; 3 ) participation was voluntary; 4) the data would be presented only in an aggregated form in professional journals; 5) they could experience the new hair washing pad, as an advantage; 6) a disadvantage was the time required to respond to the questionnaires. Informed consent was obtained. They were instructed on using the pad. Nurses did not receive any incentives to participate in this study.

Patients or legally authorized representative received a letter informing them about the study aim and methods, which included the following: 1), 3)-6) were same as that for nurses; 2) refusal to participate would not influence their care. Informed consent was obtained. Patients received a bottle of shampoo for participating in this study.

\section{Results}

As Table 1 shows, 36 nurses participated (90\% response rate) in this study. Majority of them were in their 20s $(69 \%)$ with $6 \pm 4$ years of nursing experience (mean \pm SD). All five units' demographic data were even. Majority of patients were more than 60 years of age $(82 \%)$ and men (69\%). For hair washing characteristics, hair length of less than $5 \mathrm{~cm}(63 \%), 4 \pm 2$ days since the last wash, and 1-2 liters $(60 \%)$ of used water were common. 
For evaluation of the pad (Figure 3), the overall evaluation was very good/good (91\%). Structural aspects included good water absorption $(100 \%)$, good shape $(90 \%)$, good water collection behind the neck $(83 \%)$, and good design in terms of pattern/color (72\%). Additional free comments were "good size," "colorful to feel joyful to wash but too pink for man," "heavy weight after use," and "looks like diaper." Usage aspects included easy to wash (100\%), no water leakage (95\%), no spreading of water $(94 \%)$, easy to prepare $(94 \%)$, and easy to tidy up (100\%). Additional free comments were "easy usage due to similar use as a diaper," "might be difficult in case of long hair," and "diaper might be cheaper than the developed pad." The holistic evaluation was satisfactory in both structural and usage aspects.

For comparison with diaper, majority nurses ( $84 \%$ ) did not want to use diapers, although most had experience of using them (95\%) (Figure 4). After our trial, nurses (85\%) preferred the developed pad. Reasons were "better water absorption," "better gathering behind the neck," "better size," "easier usage due to simple shape," "no ethical issue," and "no cost for patient." The integrated result was that the developed pad was better than the diaper.

Concerning patients' result, neck comfort was reported as being very comfortable/comfortable (84\%), neither (5\%), and not so comfortable (11\%). In free comments, "convenient due to quickness," "felt like usual hair washing," and "surprised to have hair washing in bed" were reported.

Table 1. Demographic data and hair washing characteristic $(\mathrm{N}=36)$

\begin{tabular}{|c|c|c|c|c|}
\hline Contents & & Number & $(\%)$ & Mean \pm SD \\
\hline \multicolumn{5}{|l|}{ Nurse } \\
\hline \multirow[t]{2}{*}{ Age } & $20 \mathrm{~s}$ & 25 & (69) & \\
\hline & $30 \mathrm{~s}$ & 11 & $(31)$ & \\
\hline \multirow[t]{4}{*}{ Nursing experience } & Less than 5 years & 18 & (50) & $6 \pm 4$ years \\
\hline & $6-10$ years & 11 & (31) & \\
\hline & $11-15$ years & 4 & (11) & \\
\hline & More than 15 years & 3 & (8) & \\
\hline \multirow[t]{2}{*}{ Department } & Intensive care unit & 18 & $(50)$ & \\
\hline & Ward & 18 & $(50)$ & \\
\hline \multicolumn{5}{|l|}{ Patient } \\
\hline \multirow[t]{2}{*}{ Sex } & Women & 11 & $(31)$ & \\
\hline & Men & 25 & (69) & \\
\hline \multirow[t]{6}{*}{ Age $(n=33)$} & $30 \mathrm{~s}$ & 2 & (6) & $65 \pm 15$ years old \\
\hline & $40 \mathrm{~s}$ & 1 & (3) & \\
\hline & $50 \mathrm{~s}$ & 3 & (9) & \\
\hline & $60 \mathrm{~s}$ & 11 & $(34)$ & \\
\hline & $70 \mathrm{~s}$ & 9 & $(27)$ & \\
\hline & More than 80 years olds & 7 & $(21)$ & \\
\hline \multicolumn{5}{|l|}{ Hair washing characteristic } \\
\hline \multirow[t]{4}{*}{ Hair length $(\mathrm{n}=35)$} & Less than $5 \mathrm{~cm}$ & 22 & $(63)$ & \\
\hline & $5 \mathrm{~cm}$ to chin length & 9 & $(26)$ & \\
\hline & Chin to shoulder length & 3 & (9) & \\
\hline & More than shoulder length & 1 & (3) & \\
\hline Period from last wash & & & & $4 \pm 2$ days \\
\hline Used water volume & Less than 1 litter & 9 & (26) & \\
\hline \multirow[t]{3}{*}{$(\mathrm{n}=35)$} & 1-2litter & 21 & $(60)$ & \\
\hline & 2-3 litter & 5 & (14) & \\
\hline & More than 3 litter & 0 & (0) & \\
\hline
\end{tabular}




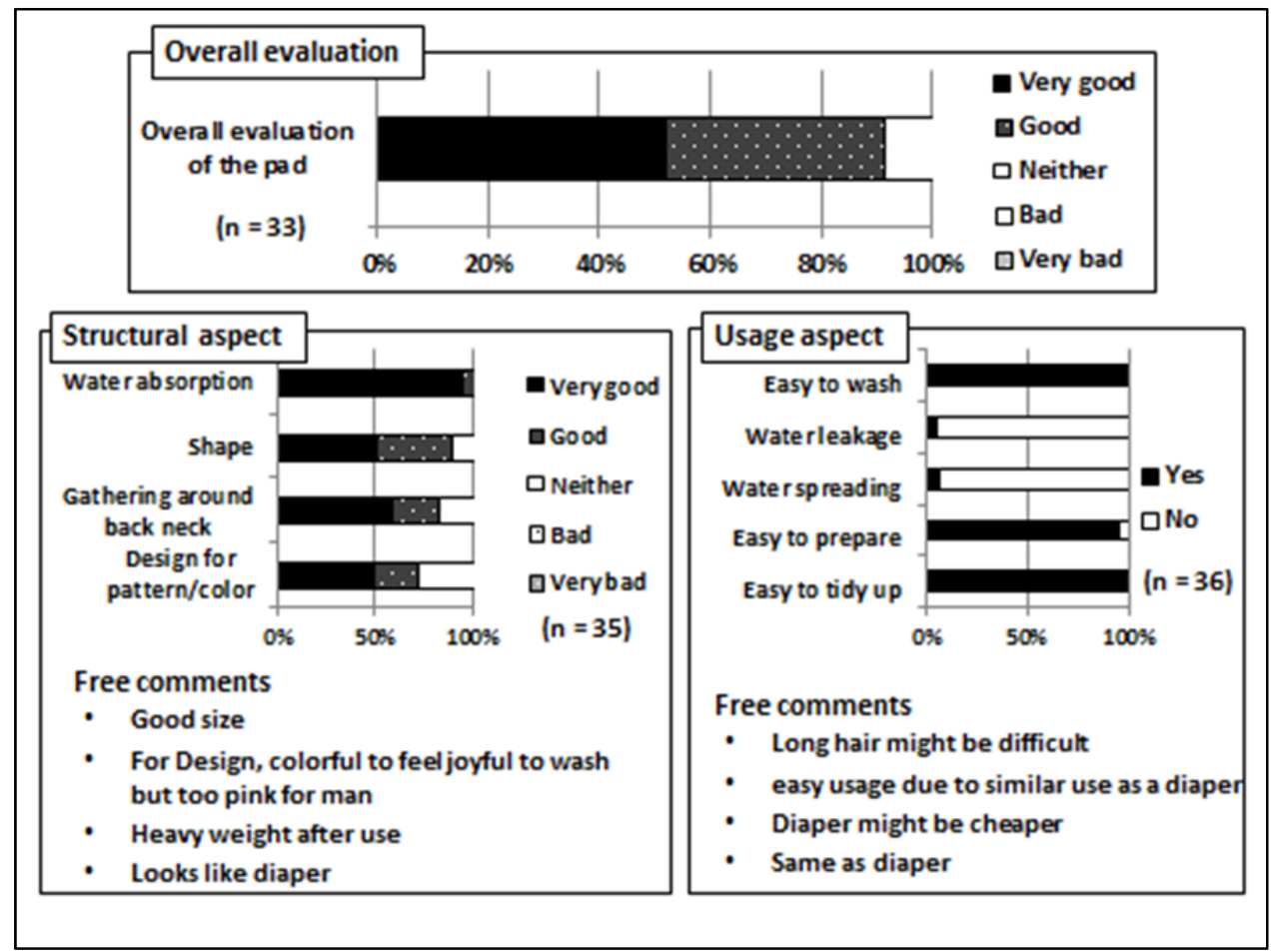

Figure 3. Joint display for evaluation of the pad

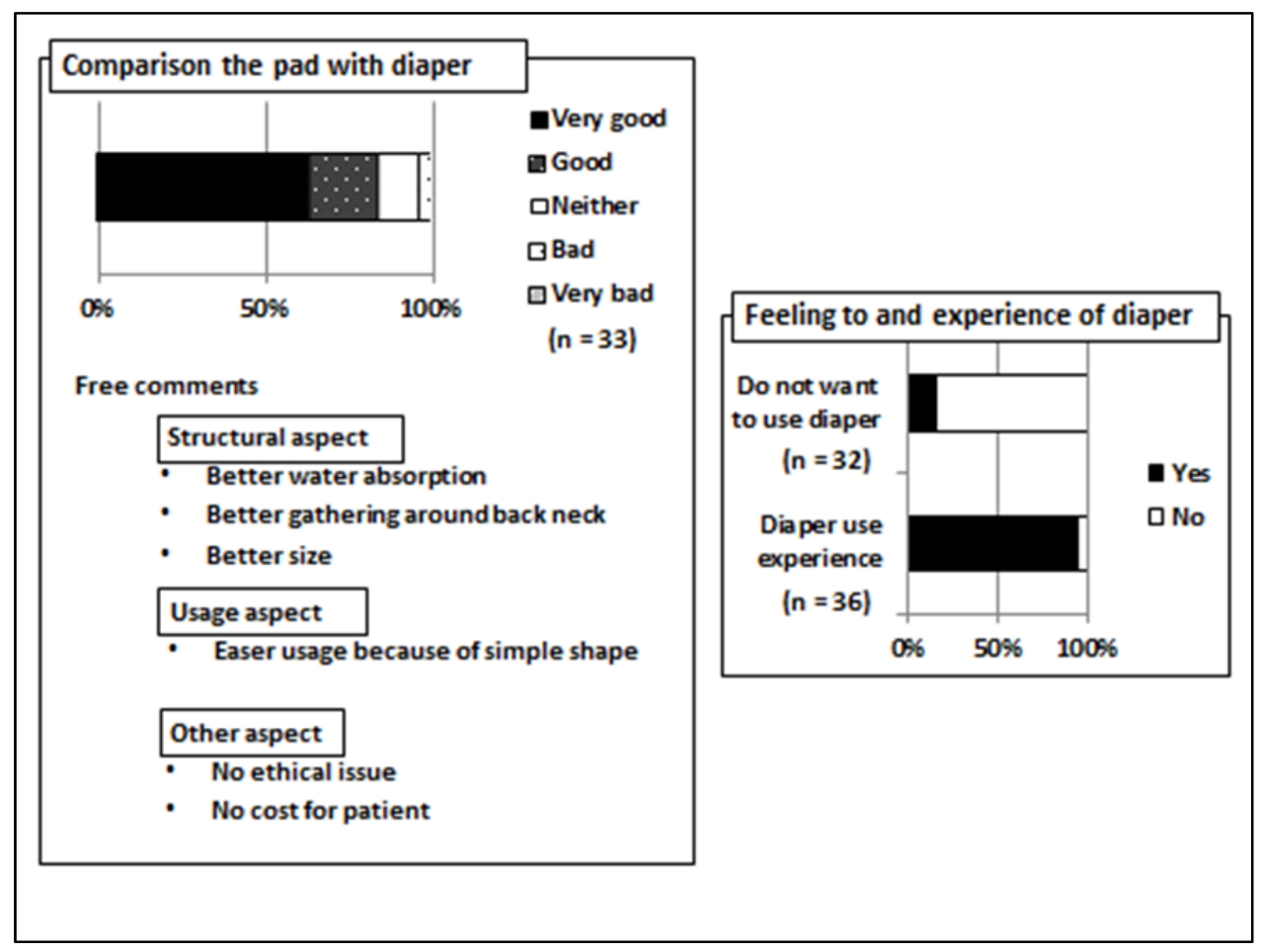

Figure 4. Joint display for comparing the pad with diaper

\section{Discussion}

Our industrial-academia-clinical team developed a disposable hair washing pad for bedridden patients. The clinical trial was conducted using a cross-sectional survey with mixed methods research. Efficient performance of 
the developed pad was established. On comparing with experience of diaper use, greater performance and relief from ethical issues were found. Challenges were the design of pattern/color, heavy weight after use, and looking like a diaper.

Our pad is precisely designed for hair washing in bed. Water-absorbing polymer is located in certain parts in the amount necessary to contain the hair washing water. Adhesive strength is also high enough to hold polymer during hair washing (Makabe et al., 2017b). Diapers' water-absorption and adhesive strength are suitable for urine volume and urination point. Gathering shape and size are also planned especially for the curve of the back of the neck and not for that of the hip, as in case of diapers. Hence, the developed pad demonstrates high performance. Furthermore, simple shape and easy usage (familiarity due to similarity with diaper) also promotes nurse acceptance. Bed bath or hair washing in bed could be an unpleasant experience for patients (Lopes, Nogueira-Martins, \& de Barros, 2013). Research on developing nursing equipment needs to be focused more on patient comfort. While body wash transits dry wash without water these days (Groven et al., 2017), using water for hair wash is important to avoid lice, because the scalp/hair gets dirty easily.

Bedridden patients tend to be critically ill and the space around the head is limited due to treatment devices for respiration, biological monitors, or many lines for infusion etc. (Eigsti, 2011; Sartini et al., 2018). Neck joint flexibility is also restricted due to stiffness. The developed pad allows for patient comfort through low time consumption, not requiring the head to be lifted high, and not requiring a change in patients' position and space ecology. Our results are comparable to the findings of recent research (Sato, Kodo, Fukushi, \& Isomoto, 2014) that hospital inpatients receive hair washing infrequently. Originally of our pad are gathering around back neck and no water accumulation at the back head due to sliding pad. Existing hair washing equipment like Kelly pad or hair washing machine are bulky and time consuming. However, our developed pad provides stress-free use in terms of preparation, equipment usage, and cleaning, which allow frequent hair washing. Furthermore, infection control is also achieved as the developed pad is disposable.

Diapers are used for elimination care, and hence placing it under the head is an ethical problem, even if unused diapers are employed. Nurses' reluctance in using diapers is reflected in findings of the recent research, although some nurses are comfortable in using diaper because of being habituated to it. As nurses' professional commitment and its effect on patient care is not correlated with years of expertise (Al-Hamdam, Dalky, \& Al-Ramadneh, 2018), novice nurses have better flexibility to adopt new idea/device than do experts. Although the developed pad might still look like a diaper, it is not a diaper. Nurses' experience of using diapers might cause a memory bias such as beliefs like diaper is useful and that they are habituated to using diapers. In Japan, patients are required to buy diapers themselves during hospitalization. Using diapers for patients' hair washing affects them financially. In addition, diaper use for hair washing is not evidence-based. Care procedures need to be easier and standardized for the highly demanding hospital environments.

The pale pink check pattern was intended to make it enjoyable for nurses, similar to being in a picnic and avoiding the appearance of a diaper. Our research team predominantly included women. Consequently, the color chosen was pink. However, a neutral design is essential for improvement, because gender features exist in both nurse and patient. Good water absorption leads to heavy weight after use. The diaper compressor is essential to reduce disposing cost and for being environmentally friendly. Moreover, shower bottle can control water pressure. This type of bottle is crucial to avoid water spreading, because the developed pad is solid even though it has good water absorption.

An implication for practice is that in patients' recovery, hair washing in bed becomes a pleasant experience (Feo \& Kitson, 2016). The developed pad contributes to nurses' hair washing care involving not just patient comfort but also ease of use (Tamura et al., 2013). In community settings, because diaper is supported by subsidies from the local government, diapers are easily used even for hair washing. Hopefully, our developed pad will be in the market in the near future and used in community settings and not just in hospital settings. Furthermore, for developing nursing equipment, industry-academia-clinical collaboration is truly essential, because our project is surely focused on achieving ideal standards.

In terms of limitations, the sample size was slightly small due to the minority patient group. Nevertheless, the size was large enough to identify performance and challenges for further improvement. Patients in this study were also predominantly men with short hair. Although patients included those with long hair in this study, there is no remarkable issue in relation to long hair. For financial matters, this time of developed pad was handmade for this study; for further stages of manufacturing, the cost needs to be reduced to be lower than the existing hair washing sheet or diapers to put our developed pad on the market. Finally, for a mixed methods research, qualitative data were weak. Although participants might have hesitated to provide written comments compared to those in an 
interview, certainly core data is corrected. Mixed methods research allowed us to evaluate the developed pad from various aspects. Details of improving the characteristics for the future are highlighted. For the future, a nationwide validation study on mega production validation is needed to reduce the cost. Hopefully, the disposable hair washing pad for bedridden patients will be in the market soon.

\section{Conclusion}

The disposable hair washing pad developed particularly for bedridden patients showed high performance in terms of water absorption, no water leakage, and easy usage. Comparing the developed pad with diapers revealed its greater performance and that it resolved ethical issues. Further manufacturing challenges need to be identified for mega production.

\section{Acknowledgements}

We sincerely thank all of the patients and nurses who participated in this study. We also thank Dr. Yukihiro Nakano (Vice president of R \& D-Development Research-Sanitary Product Research, Kao Corporation), Ms. Harumi Nagatani (Kao Corporation), Mr. Hiroshi Kadoya (Former patent agent, Akita University), Prof. Mitsutoshi Jikei (Materials Science, Akita University), Prof. Fumio Yamamoto (President of Akita University), Prof. Hideaki Andoh (Akita University) and Assistant Prof. Yuko Akagawa (Akita University). We also thank for industrial-academia department from Akita University to support our industrial-academia collaborative project. This study was funded by Akita University and Kao Corporation.

\section{Competing Interests Statement}

The authors declare that there are no competing or potential conflicts of interest.

\section{References}

Al-Hamdam, Z., Dalky, H., \& Al-Ramadneh, J. (2018). Nurses' professional commitment and its effect on patient safety. Global Journal of Health Schience, 10(1), 111-119. https://doi.org/10.5539/gihs.v10n1p111

Chen, B. K., Jalal, H., Hashimoto, H., Suen, S. C., Eggleston, K., Hurley, M., . . B Bhattacharya, J. (2016). Forecasting Trends in Disability in a Super-Aging Society: Adapting the Future Elderly Model to Japan. The Journal of the Economics of Ageing, 8, 42-51. https://doi.org/10.1016/j.jeoa.2016.06.001

Creamer, E. G. (2018). An tntroduction to fully integrated mixed methods research. Blacksburg, USA.

Dougherty, L., \& Lster, S. (2004). Clinical Nursing Procedure, Personal hygiene: Skin Care (pp. 580-586). Blackwell publishing, UK.

Eigsti, J. E. (2011). Innovative solutions: beds, baths, and bottoms: a quality improvement initiative to standardize use of beds, bathing techniques, and skin care in a general critical-care unit. Dimensions of Criticat Care Nursing, 30(3), 169-176. https://doi.org/10.1097/DCC.0b013e31820d25b1

Feo, R., \& Kitson, A. (2016). Promoting patient-centred fundamental care in acute healthcare systems. International Journal of Nursing Studies, 57, 1-11. https://doi.org/10.1016/j.ijnurstu.2016.01.006

Fetters, M. D., Curry, L. A., \& Creswell, J. W. (2013). Achieving integration in mixed methods designs-principles and practices. Health Services Research Journal, 48(6 Pt 2), 2134-2156. https://doi.org/10.1111/1475-6773.12117

Friganovic, A., Kovacevic, I., Ilic, B., Zulec, M., Kriksic, V., \& Grgas Bile, C. (2017). Healthy Settings in Hospital - How to Prevent Burnout Syndrome in Nurses: Literature Review. Acta Clinica Croatica, 56(2), 292-298. https://doi.org/10.20471/acc.2017.56.02.13

Groven, F. M., Zwakhalen, S. M., Odekerken-Schroder, G., Joosten, E. J., \& Hamers, J. P. (2017). How does washing without water perform compared to the traditional bed bath: a systematic review. BMC Geriatrics, 17(1), 31. https://doi.org/10.1186/s12877-017-0425-4

John, O. P., \& Gross, J. J. (2004). Healthy and unhealthy emotion regulation: personality processes, individual differences, and life span development. Journal of Personality, 72(6), 1301-1333. https://doi.org/10.1111/j.1467-6494.2004.00298.x

Korstjens, I., \& Moser, A. (2018). Series: Practical guidance to qualitative research. Part 4: Trustworthiness and publishing. European Journal of General Practice, 24(1), 120-124. https://doi.org/10.1080/13814788.2017.1375092

Lopes, J. L., Nogueira-Martins, L. A., \& de Barros, A. L. (2013). Bed and shower baths: Comparing the perceptions of patients with acute myocardial infarction. Journal of Clinical Nursing, 22(5-6), 733-740. 
https://doi.org/10.1111/j.1365-2702.2012.04320.x

Makabe, S., Fujita, K., Makimoto, K., Kozuka, K., Miura, M., Kanno, T., . . Mawatari, M. (2015). Impact of Japanese lifestyle on quality of life for patients with total hip arthroplasty. Journal of Physical Therapy and Health Promotion, 2(4), 51-56. https://doi.org/10.18005/PTHP0204001

Makabe, S., Izumori, S., Konno, E., Sato, Y., Suda, N., Shirakawa, H., . . Ando, K. (2017a). Development and evaluation for two types of disposable hair wash pads: Convergence mix methods. Mixed methods international reserach association Asia regional conference, Osaka, Japan, Ritsumeikan Univeristy

Makabe, S., Konno, E., Suda, N., Ikeda, Y., Shirakawa, H., Maeda, K., . . Ando, K. (2017b). Absorbent hair washing pad and its set for bedridden patients. Japan Patent Office (P6453937), 1-21. [In Japanese]

Murayama, Y. (2016). Innovation in Neurosurgery: Intellectual Property Strategy and Academia/Industrial $\begin{array}{lllll}\text { Collaboration. Neurological } & \text { Medico }\end{array}$ https://doi.org/10.2176/nmc.st.2016-0078

Nakai, Y., Hikichi, M., Hashimoto, Y., Furuta, M., \& Okamoto, S. (2009). An attempt to make a shampoo tool using paper-made diaper pads. Tottori rinsyokagaku kennkyuukaishi, 1(2), 325-331. [In Japanese]

Roberts, N. A., Levenson, R. W., \& Gross, J. J. (2008). Cardiovascular costs of emotion suppression cross ethnic $\begin{array}{lllll}\text { lines. International Journal of Psychophysiology } & 70(1), & 82-87 .\end{array}$ https://doi.org/10.1016/j.jpsycho.2008.06.003

Schlatter, M. C., \& Cameron, L. D. (2010). Emotional suppression tendencies as predictors of symptoms, mood, and coping appraisals during AC chemotherapy for breast cancer treatment. Annals of Behavioral Medicine, 40 (1), 15-29. https://doi.org/10.1007/s12160-010-9204-6

Sartini, C., Lomivorotov, V., Pieri, M., Delgado, J. C. L., Baiardo Redaelli, M., Hajjar, L., . . Landoni, G. (2018). A Systematic Review and International Web-Based Survey of Randomized Controlled Trials in the Perioperative and Critical Care Setting: Interventions Reducing Mortality. Journal of Cardiothoracic Vascular Anesthesia. https://doi.org/10.1053/j.jvca.2018.11.026

Sato, A., Kodo, Y., Fukushi, N., \& Isomoto, A. (2014). Research on the practicality of the "Shampoo Sheet" newly developed. Bulletin of Hirosaki University of Health and Welfare, 5(1), 69-75. [In Japanese]

Tamura, Y., Imanishi, S., Eto, N., Kuroki, M., Okutsu, F., \& Yamada, T. (2013). Questionnaire survey for hair washes equipment development. Bulletin of Kyoto Municipal Junior College of Nursing, (37), 59-65. [In Japanese]

Tsuruya, N., Kawashima, T., Shiozuka, M., Nakanishi, Y. \& Sugiyama, D. (2018). Academia-industry Cooperation in the Medical Field: Matching Opportunities in Japan. Clinical Therapeutics, 40(11), 1807-1812. https://doi.org/10.1016/j.clinthera.2018.10.010

\section{Copyrights}

Copyright for this article is retained by the author(s), with first publication rights granted to the journal.

This is an open-access article distributed under the terms and conditions of the Creative Commons Attribution license (http://creativecommons.org/licenses/by/4.0/). 